\title{
Ligelizumab for Chronic Spontaneous Urticaria
}

\author{
M. Maurer, A.M. Giménez-Arnau, G. Sussman, M. Metz, D.R. Baker, A. Bauer, \\ J.A. Bernstein, R. Brehler, C.-Y. Chu, W.-H. Chung, I. Danilycheva, C. Grattan, \\ J. Hébert, C. Katelaris, M. Makris, R. Meshkova, S. Savic, R. Sinclair, K. Sitz, \\ P. Staubach, B. Wedi, J. Löffler, A. Barve, K. Kobayashi, E. Hua, T. Severin, \\ and R. Janocha
}

ABSTRACT

\section{BACKGROUND}

In the majority of patients with chronic spontaneous urticaria, most currently available therapies do not result in complete symptom control. Ligelizumab is a nextgeneration high-affinity humanized monoclonal anti-IgE antibody. Data are limited regarding the dose-response relationship of ligelizumab and the efficacy and safety of ligelizumab as compared with omalizumab and placebo in patients who have moderate-to-severe chronic spontaneous urticaria that is inadequately controlled with $\mathrm{H}_{1}$-antihistamines at approved or increased doses, alone or in combination with $\mathrm{H}_{2}$-antihistamines or leukotriene-receptor antagonists.

\section{METHODS}

In a phase $2 \mathrm{~b}$ dose-finding trial, we randomly assigned patients to receive ligelizumab at a dose of $24 \mathrm{mg}, 72 \mathrm{mg}$, or $240 \mathrm{mg}$, omalizumab at a dose of $300 \mathrm{mg}$, or placebo, administered subcutaneously every 4 weeks for a period of 20 weeks, or a single 120-mg dose of ligelizumab. Disease symptoms of hives, itch, and angioedema were monitored by means of weekly activity scores. The main objective was to determine a dose-response relationship for the complete control of hives (indicated by a weekly hives-severity score of 0 , on a scale from 0 to 21 , with higher scores indicating greater severity); the primary end point of this response was assessed at week 12 . Complete symptom control was indicated by a weekly urticaria activity score of 0 (on a scale from 0 to 42 , with higher scores indicating greater severity). Safety was analyzed throughout the trial.

\section{RESULTS}

A total of 382 patients underwent randomization. At week 12, a total of $30 \%, 51 \%$, and $42 \%$ of the patients treated with $24 \mathrm{mg}, 72 \mathrm{mg}$, and $240 \mathrm{mg}$, respectively, of ligelizumab had complete control of hives, as compared with $26 \%$ of the patients in the omalizumab group and no patients in the placebo group. A dose-response relationship was established. At week 12, a total of 30\%, $44 \%$, and $40 \%$ of the patients treated with $24 \mathrm{mg}, 72 \mathrm{mg}$, and $240 \mathrm{mg}$, respectively, of ligelizumab had complete control of symptoms, as compared with $26 \%$ of the patients in the omalizumab group and no patients in the placebo group. In this small and short trial, no safety concerns regarding ligelizumab or omalizumab emerged.

\section{CONCLUSIONS}

A higher percentage of patients had complete control of symptoms of chronic spontaneous urticaria with ligelizumab therapy of $72 \mathrm{mg}$ or $240 \mathrm{mg}$ than with omalizumab or placebo. (Funded by Novartis Pharma; ClinicalTrials.gov number, NCT02477332.)
The authors' full names, academic degrees, and affiliations are listed in the Appendix. Address reprint requests to Dr. Maurer at the Department of Dermatology and Allergy, Charité-Universitätsmedizin Berlin, Charitéplatz 1, 10117 Berlin, Germany, or at marcus.maurer@charite.de.

N Engl J Med 2019;381:1321-32. DOI: 10.1056/NEJMoa1900408 Copyright @ 2019 Massachusetts Medical Society. 
HRONIC SPONTANEOUS URTICARIA IS A skin disorder that is characterized by the occurrence of itchy wheals (hives), angioedema, or both for 6 weeks or more in the absence of specific external stimuli. ${ }^{1,2}$ Studies have indicated that chronic spontaneous urticaria has a prevalence of approximately 1 case per 200 persons in adult European populations. ${ }^{3-5}$ Evidence suggests that chronic spontaneous urticaria has a long duration ${ }^{6,7}$ and can have a negative effect on quality of life. ${ }^{6,8}$ The pathogenesis of chronic spontaneous urticaria is not fully clear, but it is known to involve autoimmune mechanisms in most patients by means of IgG autoantibodies against the high-affinity receptor for the Fc region of IgE (FceRI) or less frequently against IgE and IgE autoantigens. ${ }^{9-13}$

Standard first-line treatment of chronic spontaneous urticaria consists of nonsedating (secondgeneration) $\mathrm{H}_{1}$-antihistamines at locally approved doses, with escalation up to four times the approved dose used off-label as second-line treatment; even so, the disease is still uncontrolled in some patients. ${ }^{1,14,15}$ The revised guidelines regarding the treatment of urticaria from the European Academy of Allergology and Clinical Immunology (EAACI), the Global Allergy and Asthma European Network (GA ${ }^{2} \mathrm{LEN}$ ), the European Dermatology Forum (EDF) and the World Allergy Organization (WAO ${ }^{1}$ recommend the use of omalizumab as add-on third-line therapy to $\mathrm{H}_{1}$-antihistamines. The goal of therapy, as noted in the current treatment guidelines, is complete control of the disease, yet some patients have symptoms that remain uncontrolled with the current standard of care. ${ }^{1}$ To date, add-on therapy with omalizumab has been the most effective treatment in patients who continue to have symptoms while they are taking $\mathrm{H}_{1}$-antihistamines, ${ }^{16-18}$ but some patients do not have a response, and better treatment options are needed. ${ }^{19-22}$

Ligelizumab is a new high-affinity humanized monoclonal anti-IgE antibody that has previously shown dose-dependent and time-dependent suppression of free IgE, basophil FceRI, basophil surface IgE, and skin-prick test responses to allergen that was superior in extent and duration to that observed with omalizumab. ${ }^{23}$ In a phase 2b trial, we examined the efficacy and safety of ligelizumab as compared with omalizumab and placebo in patients with chronic spontaneous urticaria that was inadequately controlled with standard-of-care therapy including $\mathrm{H}_{1}$-antihistamines.

\section{METHODS}

\section{TRIAL DESIGN AND OVERSIGHT}

This phase $2 \mathrm{~b}$, dose-finding, multicenter, randomized, double-blind, active-controlled and placebocontrolled trial was designed to establish a dose-response relationship for ligelizumab and to evaluate its efficacy and safety as compared with omalizumab and placebo (Fig. 1). The trial consisted of a screening period (days -14 to 1 ), a treatment period (days 1 to 140 [20 weeks]), and a follow-up period after the cessation of the trial regimens (days 141 to 309 [24 weeks]). Patients who remained in the follow-up period for at least 12 weeks and had active disease (defined as a mean weekly urticaria activity score [measured twice daily] of $\geq 12$; scores range from 0 to 42 , with higher scores indicating greater severity; minimally important difference [MID], 9.5 to 10.0 points $)^{24-27}$ were eligible to enter an extension study (ClinicalTrials.gov number, NCT02649218) from week 32 onward. The trial protocol, with the statistical analysis plan, is available with the full text of this article at NEJM.org.

This trial was designed and sponsored by Novartis Pharma. The institutional review board at each participating center approved the protocol. Patients provided written informed consent before any assessment was performed. Data were collected by the trial investigators according to Good Clinical Practice guidelines and were analyzed by the sponsor. The first draft of the manuscript was written by a medical writer paid by the sponsor, with critical input and approval from all authors. All the authors critically reviewed each manuscript draft, provided substantial input on the content, and made the decision to submit the manuscript for publication. The authors vouch for the accuracy and completeness of the data and for the fidelity of the trial to the protocol.

\section{PATIENTS}

Eligible patients were 18 to 75 years of age and had chronic spontaneous urticaria that was inadequately controlled with $\mathrm{H}_{1}$-antihistamines at 


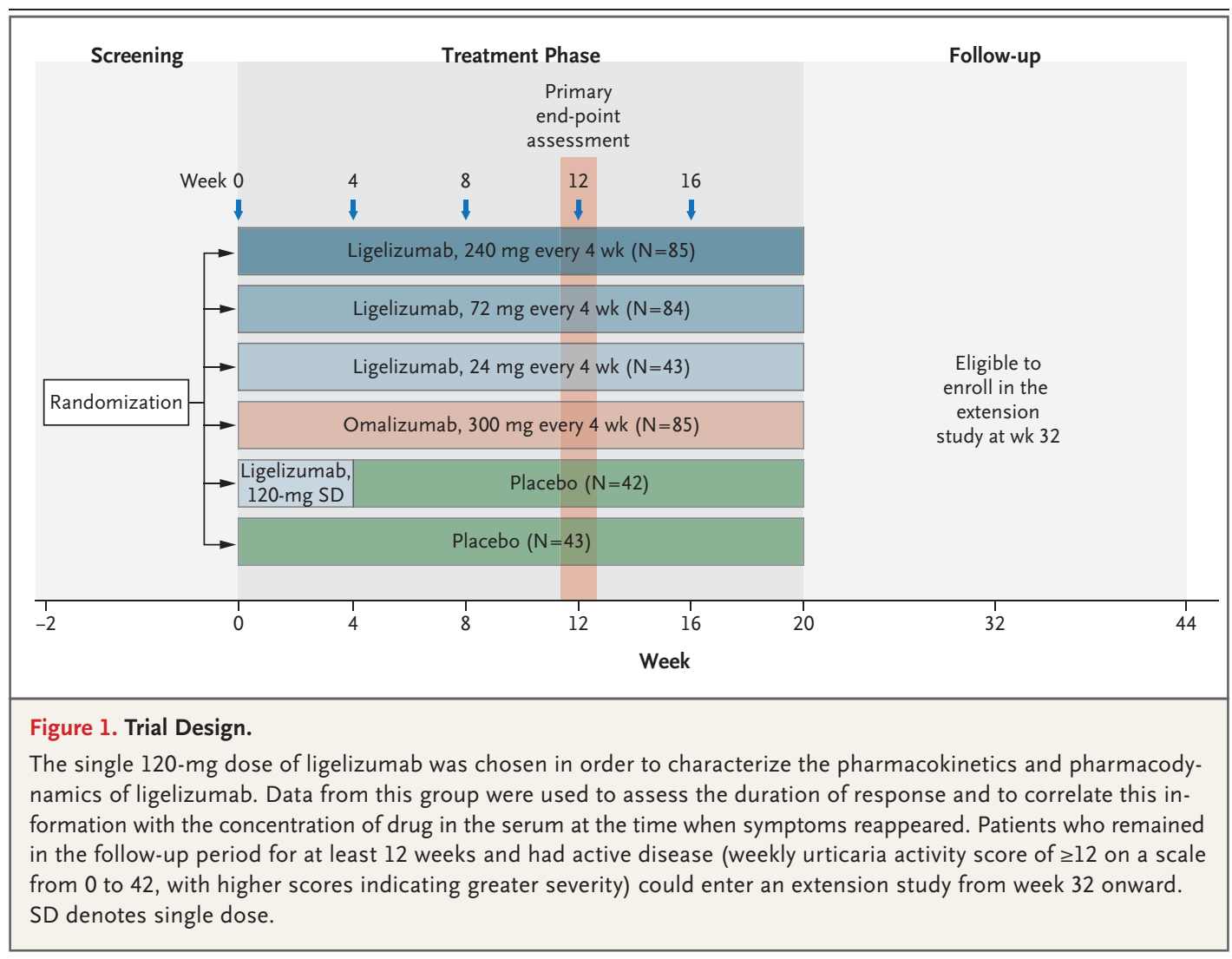

approved or increased doses alone or in combination with $\mathrm{H}_{2}$-antihistamines or leukotrienereceptor antagonists. Key inclusion criteria were the following: mild-to-moderate chronic spontaneous urticaria, defined as a weekly urticaria activity score of at least 16 (disease activity categories are as follows: 0 , symptom free; 1 to 6 , well-controlled urticaria; 7 to 15 , mild urticaria; 16 to 27 , moderate urticaria; and 28 to 42 , severe urticaria $\left.{ }^{26}\right)$; a weekly hives-severity score of at least 8 (scores range from 0 to 21, with higher values indicating greater severity; MID, 5.0 to 5.5 points) ${ }^{27}$ during the 7 days before randomization (day 1); and an in-clinic urticaria activity score of at least 4 (scores range from 1 to 6 , with higher values indicating greater severity; MID, not available) on at least one of the screening visit days. Key exclusion criteria were the following: previous exposure to omalizumab or ligelizumab, any other skin disease that is associated with chronic itching that might confound the trial evaluations and results, and a clearly defined underlying cause of chronic urticaria other than chronic spontaneous urticaria (e.g., inducible urticaria).

The demographic and clinical characteristics of the patients and the disease activity were elicited or measured at baseline. The Chronic Urticaria Index (CU Index; Viracor-IBT Laboratories), an in vitro basophil histamine-release assay in which a serum sample obtained from a patient is mixed with donor basophils and the released histamine levels are measured by a quantitative enzyme immunoassay, was performed at baseline. A positive CU Index (scores range from 1 to 50 , with scores $\geq 10$ representing a positive result) indicates that a patient has either an autoimmune basis for the urticaria or an alternative histamine-releasing factor that has been associated with greater disease severity than that in patients with a negative CU Index. ${ }^{28,29}$ The serum samples for this analysis were obtained centrally and analyzed by Viracor-IBT Laboratories. From the time that samples were obtained until analysis, they were kept frozen to ensure sample integrity. 


\section{TRIAL PROCEDURES}

Patients were randomly assigned, in a 2:2:2:1:1:1 ratio, to receive one of the following trial regimens: $240 \mathrm{mg}$ of ligelizumab every 4 weeks, $72 \mathrm{mg}$ of ligelizumab every 4 weeks, $300 \mathrm{mg}$ of omalizumab every 4 weeks, $24 \mathrm{mg}$ of ligelizumab every 4 weeks, placebo every 4 weeks, or a single 120-mg dose of ligelizumab at week 0 followed by placebo every 4 weeks; the treatment period was 20 weeks (Fig. 1). The single 120-mg dose of ligelizumab was used to gain blinded washout information, to determine the level of ligelizumab in serum that was associated with the return of itch and hives symptoms, and to identify an appropriate administration interval for phase 3 trials.

Adjustments to the doses of trial agents were not permitted. Nonsedating $\mathrm{H}_{1}$-antihistamines were used as background medication and as rescue medication as needed during the screening, treatment, and follow-up periods. This trial required concurrent use of $\mathrm{H}_{1}$-antihistamines (at locally approved doses or at increased doses up to four times the locally approved dose) alone or in combination with $\mathrm{H}_{2}$-antihistamines or leukotriene-receptor antagonists (montelukast, zafirlukast, or pranlukast) as background medication. It was recommended that patients continue taking a stable background medication throughout the trial, and patients were not permitted to switch their rescue medications.

\section{END-POINT MEASURES}

The main objective of the trial was to establish a dose-response relationship with respect to the achievement of complete hives response (weekly hives-severity score of 0 ) at week 12 . Complete hives response was chosen as the primary end point because hives were considered to be a more objective symptom than itch and are specific to chronic spontaneous urticaria; itch is subjective and nonspecific and can be of different origins. We chose week 12 as the time point for the assessment of the primary end point because this was also the time point for the primary end point in phase 3 trials of omalizumab. ${ }^{19,20}$

Secondary end points included the following: the efficacy of specific ligelizumab doses of $24 \mathrm{mg}$, $72 \mathrm{mg}$, and $240 \mathrm{mg}$ as compared with omalizumab at a dose of $300 \mathrm{mg}$ with respect to the achievement of complete hives response at weeks 12 and 20; the efficacy of ligelizumab at doses of $24 \mathrm{mg}, 72 \mathrm{mg}$, and $240 \mathrm{mg}$ as compared with placebo and with omalizumab at a dose of $300 \mathrm{mg}$ in the mean change from baseline in the weekly hives-severity score, in the weekly itch-severity score (scores range from 0 to 21 , with higher scores indicating greater severity; MID, 4.5 to 5.0 points), in the weekly urticaria activity score, and in the weekly angioedema activity score (scores range from 0 to 105, with higher scores indicating greater severity; MID, not available); and the safety of ligelizumab as compared with placebo and with omalizumab at a dose of $300 \mathrm{mg}$ during the 20 weeks of the treatment phase and 24 weeks of follow-up.

\section{STATISTICAL ANALYSIS}

The null hypothesis of a constant dose-response curve for the primary efficacy end point was tested at a significance level of $5 \%$ against the one-sided alternative hypothesis of a nonconstant dose-response curve with the use of the multiple comparison procedure and modeling method, which indicates the potential dose-response relationship. ${ }^{30,31}$ The dose-response curve was modeled as a weighted average of prespecified model candidates with the use of bootstrap sampling. The dose-response model was derived from testing a range of ligelizumab doses, starting at $24 \mathrm{mg}$ and then $72 \mathrm{mg}$ and $240 \mathrm{mg}$, with placebo considered to be the zero dose. For the primary variable, patients who had data missing because of discontinuation before the week 12 assessment were considered not to have had a response, regardless of their last available assessment of the weekly hives-severity score.

Since the statistical analysis plan did not include a provision for correcting for multiplicity when tests were conducted for secondary or other outcomes, results are reported as point estimates with $95 \%$ confidence intervals. This was a small and clinically nondirective trial. The widths of the confidence intervals have not been adjusted for multiplicity, so the intervals should not be used to infer any effects for secondary outcomes.

\section{RESULTS}

\section{PARTICIPANTS}

A total of 574 patients were screened, of whom 382 were randomly assigned to a trial group. A 
total of 338 patients $(88 \%)$ completed the treatment phase of the trial (Fig. S1 in the Supplementary Appendix, available at NEJM.org). There were no notable imbalances among the trial groups regarding the demographic and clinical characteristics of the patients at baseline (Table 1).

\section{PRIMARY END POINT}

The main objective of the trial was achieved, with ligelizumab showing a dose-response relationship with respect to the achievement of complete hives response (weekly hives-severity score of 0 ) at week 12 (primary end point) in patients with chronic spontaneous urticaria. The dose-response curve showed a steep dose-response relationship with a plateau starting close to the 72-mg dose of ligelizumab (Fig. 2A); no further improvement in response was noted with the 240-mg dose.

\section{SECONDARY END POINTS}

At week 12 , a total of $30 \%, 51 \%$, and $42 \%$ of the patients treated with $24 \mathrm{mg}, 72 \mathrm{mg}$, and $240 \mathrm{mg}$, respectively, of ligelizumab had a complete hives response, as compared with $26 \%$ of the patients in the omalizumab group (Fig. 2B) and no patients in the placebo group. The response with the 72-mg dose of ligelizumab was maintained at week 20 , with $51 \%$ of the patients having a response, as compared with $34 \%$ of those in the omalizumab group. A total of $45 \%$ of the patients in the group that received the 240-mg dose of ligelizumab had a response. The response regarding itch severity (weekly itch-severity score of 0 ) showed a pattern similar to that seen with the weekly hives-severity score. Details are provided in Figure S2 in the Supplementary Appendix.

At week 12, a total of $30 \%, 44 \%$, and $40 \%$ of patients treated with $24 \mathrm{mg}, 72 \mathrm{mg}$, and $240 \mathrm{mg}$, respectively, of ligelizumab had complete control of symptoms (weekly urticaria activity score of 0 ), as compared with $26 \%$ of the patients in the omalizumab group and none of the patients in the placebo group (Fig. S2 in the Supplementary Appendix). At week 20, a response was observed in $39 \%$ of the patients who received the $72-\mathrm{mg}$ dose of ligelizumab and in $40 \%$ of those who received the 240-mg dose, as compared with $31 \%$ of those in the omalizumab group and 5\% of those in the placebo group. After the end of the treatment phase, the median time to loss of response in patients who had had a response at week 20 was 3 weeks, 4 weeks, and 10.5 weeks among patients who received $24 \mathrm{mg}, 72 \mathrm{mg}$, and $240 \mathrm{mg}$, respectively, of ligelizumab, as compared with 4 weeks among patients in the omalizumab group and 1 week among those in the placebo group.

The median (with interquartile ranges) and mean weekly hives-severity scores are shown in Figure 3. Patients in the groups that received the 72 -mg or 240 -mg doses of ligelizumab had median weekly hives-severity scores close to 0 from weeks 12 to 20. The mean changes from baseline to week 32 in the weekly hives-severity score, itch-severity score, and urticaria activity score are shown in Figure S3 in the Supplementary Appendix. The single 120-mg dose of ligelizumab resulted in suppression of symptoms that was similar to that seen with the 72-mg and 240-mg doses at week 4 and that lasted until week 8 . At week 12, the mean changes from baseline in the angioedema activity score were $-21.1,-37.6$, and -27.3 among patients treated with $24 \mathrm{mg}, 72 \mathrm{mg}$, and $240 \mathrm{mg}$, respectively, of ligelizumab, as compared with -23.1 in the omalizumab group and -23.6 in the placebo group (Fig. S3 in the Supplementary Appendix).

\section{SAFETY}

A higher proportion of adverse events that were considered by the investigators to be related to treatment was seen in the groups that received the 72-mg and 240-mg doses of ligelizumab than in the other groups (Table 2). These results were driven by mild or moderate injection-site reactions (in $4 \%$ of the patients in the $72-\mathrm{mg}$ group and $7 \%$ of those in the $240-\mathrm{mg}$ group) and by mild injection-site erythema (in $2 \%$ and $6 \%$, respectively). All other adverse events that were considered to be possibly related to treatment during the trial were reported in three or fewer patients per group, with no meaningful differences among the trial groups. Serious adverse events were reported in $7 \%, 2 \%$, and $2 \%$ of the patients treated with $24 \mathrm{mg}, 72 \mathrm{mg}$, and $240 \mathrm{mg}$, respectively, of ligelizumab, as compared with $4 \%$ of the patients treated with omalizumab and $9 \%$ of those who received placebo.

The most frequently reported adverse events (occurring in $\geq 10 \%$ of the patients overall) were viral upper respiratory tract infection (in 20\%), upper respiratory tract infection (in 13\%), and 


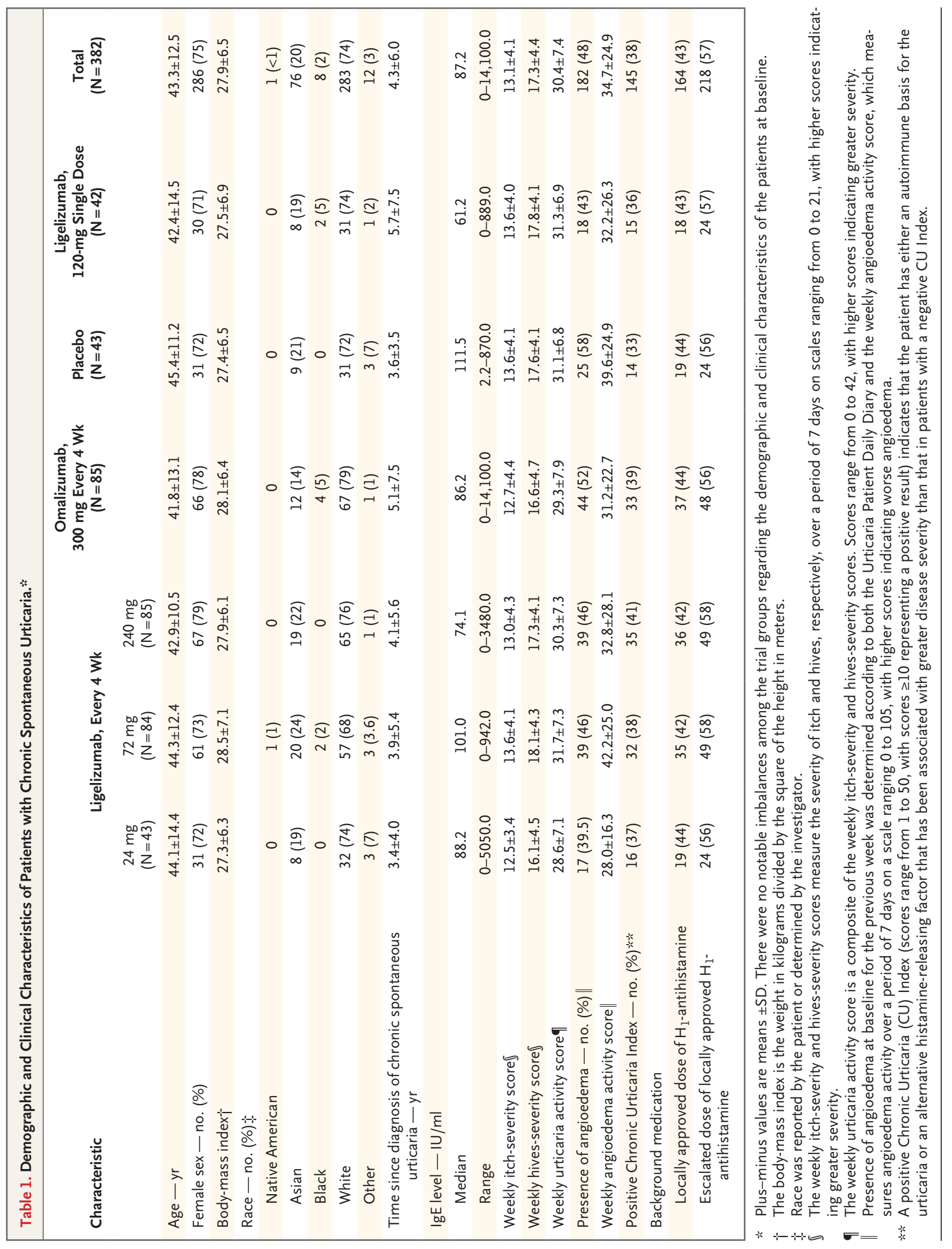




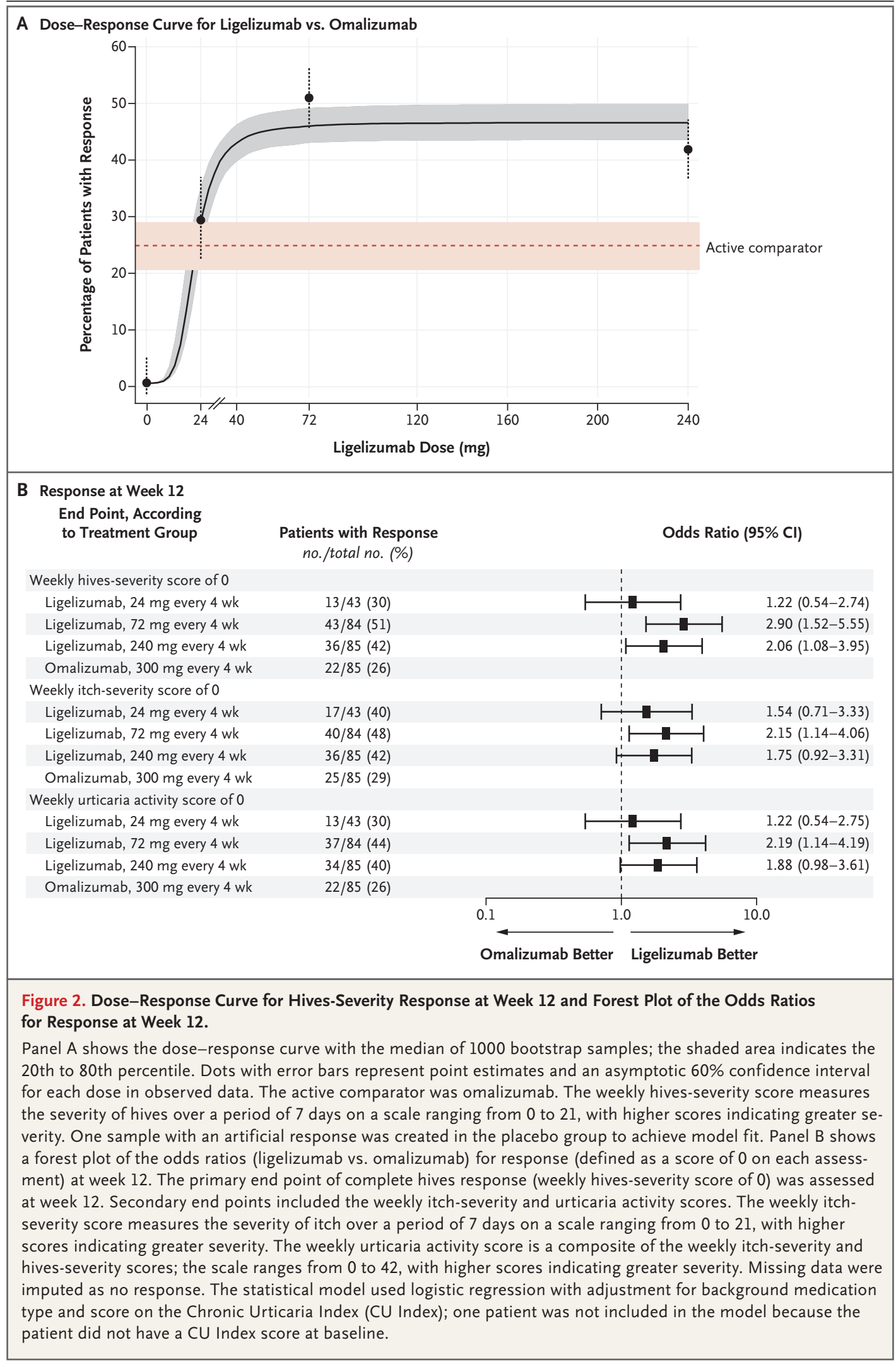

N ENGLJ MED 381;14 NEJM.ORG OCTOBER 3, 2019

The New England Journal of Medicine

Downloaded from nejm.org at UNIVERSITY OF LEEDS on October 28, 2019. For personal use only. No other uses without permission. 


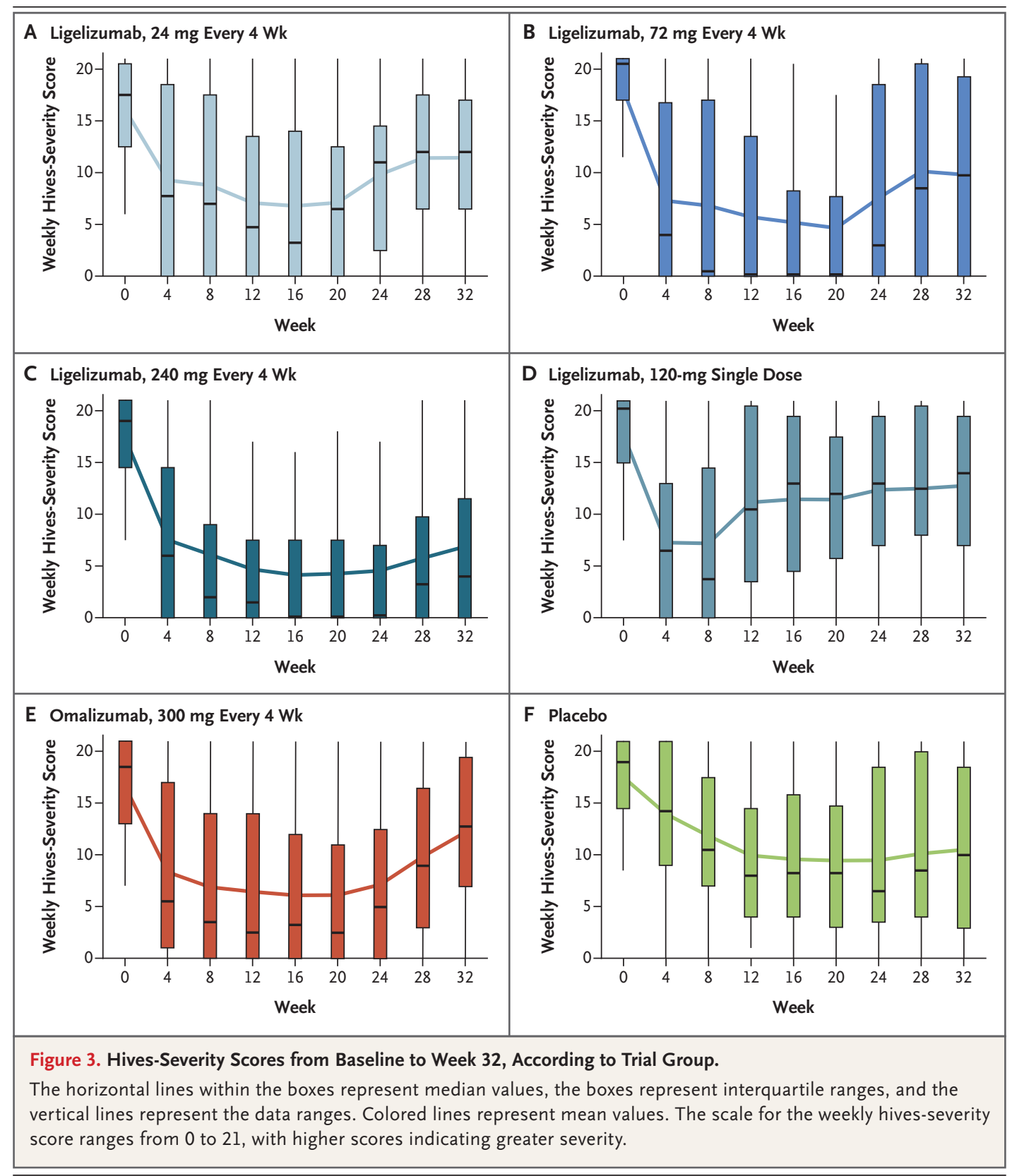

headache (in 11\%). Viral upper respiratory tract infection was reported more frequently in the placebo group (30\%) than in the four groups that received ligelizumab (15 to $24 \%$ ) or in the omalizumab group (20\%). No deaths or anaphylaxis events were reported in any of the trial groups.

\section{DISCUSSION}

Most currently available therapies for chronic spontaneous urticaria do not result in complete control of symptoms in the majority of patients. The main objective of this trial was achieved, with a clear dose-response relationship being observed with ligelizumab in the achievement of complete hives response (weekly hives-severity score of 0 ) at week 12 (primary end point) in patients with chronic spontaneous urticaria. Our trial showed that $72 \mathrm{mg}$ of ligelizumab, administered subcutaneously every 4 weeks, resulted in complete hives response in $51 \%$ of the patients, whereas $26 \%$ of the patients treated with omaliz- 


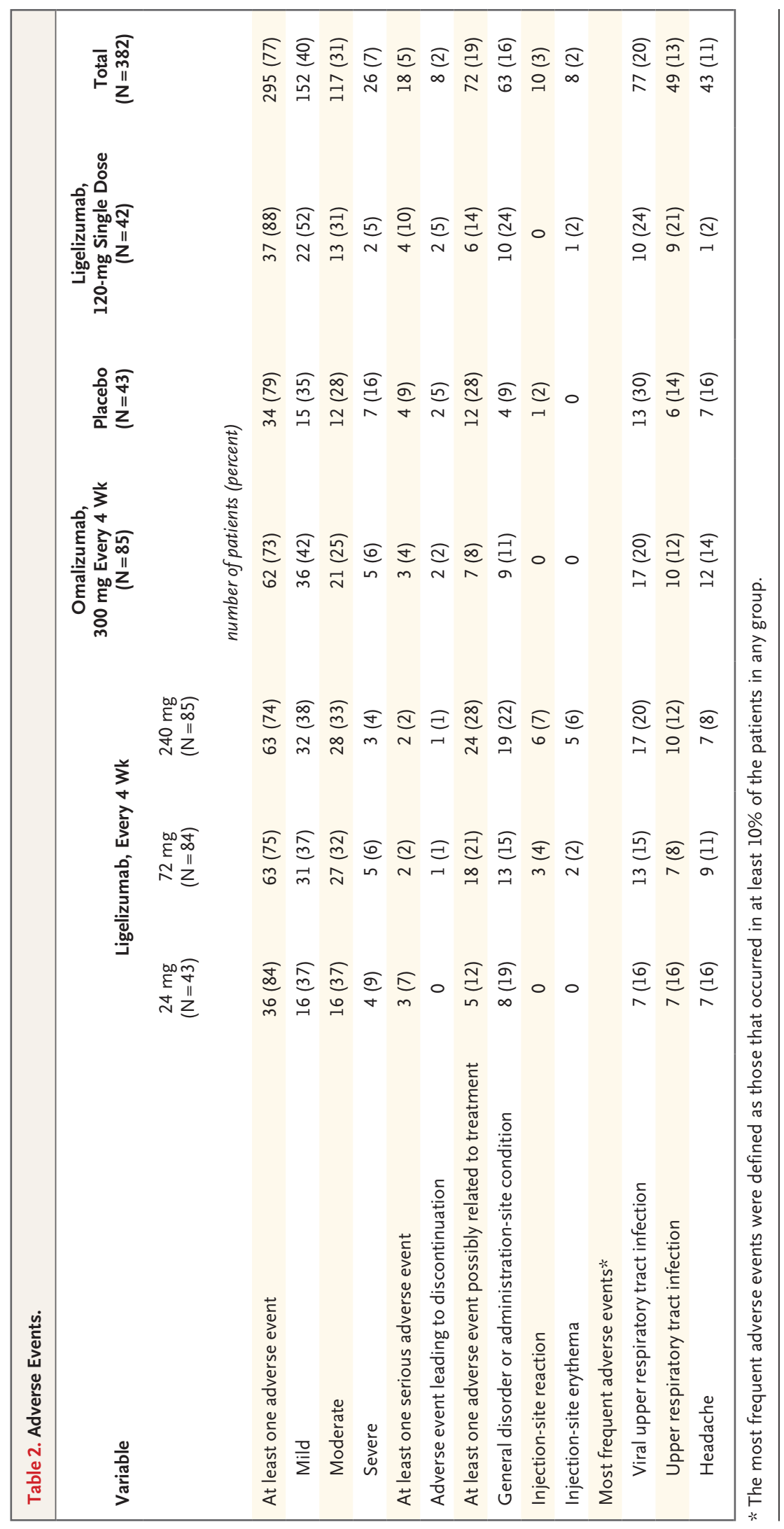

N ENGLJ MED 381;14 NEJM.ORG OCTOBER 3, 2019 
umab at a dose of $300 \mathrm{mg}$, which is the dose that has been considered to be effective in other studies, ${ }^{19-21}$ had this response.

The frequency of response according to the weekly urticaria activity score in the omalizumab group at week 12 was lower than in previous studies (34 to 44\%). ${ }^{19-21}$ An explanation for this finding may be that a higher percentage of patients in our trial had a positive CU Index (indicating an autoimmune type IIb component to their chronic spontaneous urticaria), ${ }^{28}$ had angioedema (Table 1), and had received treatment with elevated doses of $\mathrm{H}_{1}$-antihistamines or combination therapies. Previous studies have shown that a positive CU Index was associated with increased severity of chronic urticaria and that significantly more patients with refractory chronic urticaria had a positive CU Index than those who had had a response to $\mathrm{H}_{1}$-antihistamines. ${ }^{28,29}$

In the current trial, responses according to changes from baseline in the weekly hives-severity score, itch-severity score, and urticaria activity score were observed as early as week 4 after receipt of the 72-mg, 120-mg, and 240-mg doses of ligelizumab, thus indicating an onset of action within this time frame for ligelizumab. The suppression of symptoms after the single $120-\mathrm{mg}$ dose of ligelizumab was maintained until week 8 , after which symptoms returned to the level observed with placebo. In contrast, a partial relapse of symptoms was noted with the 72-mg dose of ligelizumab toward the end of the 4-week administration interval. These data suggest that although the 72-mg dose of ligelizumab led to rapid control of symptoms, a dose higher than $72 \mathrm{mg}$ could potentially provide enough drug effect throughout the administration interval to minimize relapse of symptoms and offer sustained control of symptoms throughout the 4-week administration interval. In support of this sustained treatment effect, the median time to loss of complete response in patients who had a weekly urticaria activity score of 0 at week 20 (end of the treatment phase) was greatest in the group that was treated with $240 \mathrm{mg}$ of ligelizumab (10.5 weeks) and was similar in the groups that received $72 \mathrm{mg}$ of ligelizumab or $300 \mathrm{mg}$ of omalizumab (4 weeks). Previous stud- ies of omalizumab have indicated that some patients have a return of symptoms within 4 weeks (i.e., before the administration of the next dose) ${ }^{32,33}$ Future studies will be needed to determine the dose of ligelizumab that results in a sustained clinical response throughout the administration interval.

The percentage of patients who had at least one adverse event was similar among the ligelizumab dose groups, the omalizumab group, and the placebo group. Adverse events were reported in $84 \%, 75 \%$, and $74 \%$ of the patients who were treated with $24 \mathrm{mg}, 72 \mathrm{mg}$, and $240 \mathrm{mg}$, respectively, of ligelizumab every 4 weeks and in $88 \%$ of the patients who received the single 120-mg dose of ligelizumab, $73 \%$ of those in the omalizumab group, and $79 \%$ of those in the placebo group. The higher incidence of injection-site reactions and injection-site erythema observed with ligelizumab at the doses of $72 \mathrm{mg}$ and $240 \mathrm{mg}$ than in the other groups may have been associated with differences in the administration volumes and compositions. The administration of the 240-mg dose of ligelizumab involved two injections of active drug (1.0 ml each), as compared with injections of one active drug $(0.6 \mathrm{ml})$ and one placebo $(0.6 \mathrm{ml})$ that were used for the 72-mg dose. No cases of anaphylaxis were reported in this trial.

In conclusion, in patients with moderate-tosevere chronic spontaneous urticaria, ligelizumab showed a clear dose-response relationship with regard to complete hives response (weekly hivesseverity score of 0 ) at week 12 . Ligelizumab resulted in rapid and sustained symptom control in patients with chronic spontaneous urticaria. In this small trial, side effects or laboratory abnormalities were not dose-limiting. Larger and longer trials are needed to establish the clinical efficacy of ligelizumab in patients with chronic spontaneous urticaria and its comparative profile with that of omalizumab.

A data sharing statement provided by the authors is available with the full text of this article at NEJM.org.

Supported by Novartis Pharma.

Disclosure forms provided by the authors are available with the full text of this article at NEJM.org.

We thank Martin Wallace, Ph.D., of Novartis Ireland, for providing medical writing assistance in accordance with Good Publication Practice guidelines (www.ismpp.org/gpp3). 


\section{APPENDIX}

The authors' full names and academic degrees are as follows: Marcus Maurer, M.D., Ana M. Giménez-Arnau, M.D., Ph.D., Gordon Sussman, M.D., Martin Metz, M.D., Diane R. Baker, M.D., Andrea Bauer, M.D., Jonathan A. Bernstein, M.D., Randolf Brehler, M.D., Chia-Yu Chu, M.D., Ph.D., Wen-Hung Chung, M.D., Inna Danilycheva, M.D., Clive Grattan, M.D., Jacques Hébert, M.D., Constance Katelaris, M.D., Ph.D., Michael Makris, M.D., Raisa Meshkova, M.D., D.Sc., Sinisa Savic, M.D., Ph.D., Rodney Sinclair, M.D., Karl Sitz, M.D., Petra Staubach, M.D., Bettina Wedi, M.D., Jürgen Löffler, Ph.D., Avantika Barve, Ph.D., Kenneth Kobayashi, M.D., Eva Hua, M.Sc., Thomas Severin, M.D., and Reinhold Janocha, Ph.D.

The authors' affiliations are as follows: the Department of Dermatology and Allergy, Charité-Universitätsmedizin Berlin, Berlin (M. Maurer, M. Metz), the Department of Dermatology, University Allergy Center, University Hospital Carl Gustav Carus, Technical University Dresden, Dresden (A. Bauer), the Department of Dermatology, University Hospital Münster, Münster (R.B.), the Department of Dermatology, University Medical Center Mainz, Mainz (P.S.), and the Department of Dermatology and Allergy, Comprehensive Allergy Center, Hannover Medical School, Hannover (B.W.) — all in Germany; the Dermatology Department, Hospital del Mar-Institut Hospital del Mar d'Investigacions Mèdiques, Universitat Autònoma Barcelona, Barcelona (A.M.G.-A.); the Division of Allergy and Clinical Immunology, St. Michael's Hospital and University of Toronto, Toronto (G.S.), Service d'Allergie, Centre Hospitalier Université LavalCentre Hospitalier Universitaire de Québec, Quebec, QC (J.H.), and the Department of Medicine, University of Ottawa, Ottawa (K.K.) — all in Canada; Baker Allergy Asthma and Dermatology Clinic, Portland, OR (D.R.B.); University of Cincinnati College of Medicine, Department of Internal Medicine, Division of Immunology, Rheumatology, and Allergy and Bernstein Clinical Research Center, Cincinnati (J.A.B.); the Department of Dermatology, National Taiwan University Hospital and National Taiwan University College of Medicine (C.-Y.C.), and the Department of Dermatology, Chang Gung Memorial Hospital (W.-H.C.), Taipei, Taiwan; the National Research Center-Institute of Immunology Federal Medical-Biological Agency of Russia, Moscow (I.D.), and the Department of Clinical Immunology and Allergology, Smolensk State Medical University, Smolensk (R.M.) — both in Russia; St. John's Institute of Dermatology, Guy's and St. Thomas' Hospitals NHS Foundation Trust, London (C.G.), and the National Institute for Health Research-Leeds Biomedical Research Centre and Leeds Institute of Rheumatic and Musculoskeletal Medicine, and the Department of Clinical Immunology and Allergy, St. James's University Hospital, Leeds (S.S.) — all in the United Kingdom; the School of Medicine, Western Sydney University, and the Immunology and Allergy Unit, Campbelltown Hospital, Campbelltown, NSW (C.K.), and Sinclair Dermatology and the Epworth Hospital, Melbourne, VIC (R.S.) — all in Australia; the Second Department of Dermatology and Venereology, Attikon University Hospital, Athens (M. Makris); Little Rock Allergy and Asthma Clinic, Little Rock, AR (K.S.); Novartis Pharma, Basel, Switzerland (J.L., T.S., R.J.); Novartis Pharmaceuticals, East Hanover, NJ (A. Barve, K.K.); and Shanghai Novartis Trading, Shanghai, China (E.H.).

\section{REFERENCES}

1. Zuberbier T, Aberer W, Asero R, et al. The EAACI/GA LEN/EDF/WAO guideline for the definition, classification, diagnosis and management of urticaria. Allergy 2018;73:1393-414.

2. Grattan C. The urticarias: pathophysiology and management. Clin Med (Lond) 2012;12:164-7.

3. Gaig P, Olona M, Muñoz Lejarazu D, et al. Epidemiology of urticaria in Spain. J Investig Allergol Clin Immunol 2004;14: 214-20.

4. Raciborski F, Kłak A, CzarneckaOperacz M, et al. Epidemiology of urticaria in Poland - nationally representative survey results. Postepy Dermatol Alergol 2018;35:67-73.

5. Zuberbier T, Balke M, Worm M, Edenharter G, Maurer M. Epidemiology of urticaria: a representative cross-sectional population survey. Clin Exp Dermatol 2010;35:869-73.

6. Maurer M, Weller K, Bindslev-Jensen $\mathrm{C}$, et al. Unmet clinical needs in chronic spontaneous urticaria: a GA2 ${ }^{2} \mathrm{LEN}$ task force report. Allergy 2011;66:317-30.

7. van der Valk PG, Moret G, Kiemeney LA. The natural history of chronic urticaria and angioedema in patients visiting a tertiary referral centre. Br J Dermatol 2002;146:110-3.

8. Maurer M, Abuzakouk M, Bérard F, et al. The burden of chronic spontaneous urticaria is substantial: real-world evidence from ASSURE-CSU. Allergy 2017; 72:2005-16.

9. Kaplan AP. Chronic urticaria - new concepts regarding pathogenesis and treatment. Curr Allergy Asthma Rep 2002;2: 263-4.

10. Sabroe RA, Greaves MW. Chronic idiopathic urticaria with functional autoantibodies: 12 years on. Br J Dermatol 2006; 154:813-9.

11. Kolkhir P. Autoimmune chronic spontaneous urticaria: what we know and what we do not know. J Allergy Clin Immunol 2017;139:1772-81.

12. Schmetzer O, Lakin E, Topal FA, et al. IL-24 is a common and specific autoantigen of IgE in patients with chronic spontaneous urticaria. J Allergy Clin Immunol 2018;142:876-82.

13. Altrichter S, Peter HJ, Pisarevskaja D, Metz M, Martus P, Maurer M. IgE mediated autoallergy against thyroid peroxidase - a novel pathomechanism of chronic spontaneous urticaria? PLoS One 2011;6(4):e14794.

14. Kaplan AP. Chronic spontaneous urticaria: pathogenesis and treatment considerations. Allergy Asthma Immunol Res 2017;9:477-82.

15. Guillén-Aguinaga S, Jáuregui Presa I, Aguinaga-Ontoso E, Guillén-Grima F, Ferrer M. Updosing nonsedating antihis- tamines in patients with chronic spontaneous urticaria: a systematic review and meta-analysis. Br J Dermatol 2016;175: 1153-65.

16. Curto-Barredo L, Spertino J, FiguerasNart I, et al. Omalizumab updosing allows disease activity control in patients with refractory chronic spontaneous urticaria. Br J Dermatol 2018;179:210-2.

17. Zhao ZT, Ji CM, Meng L, et al. Omalizumab for the treatment of chronic spontaneous urticaria: a meta-analysis of randomized clinical trials. J Allergy Clin Immunol 2016;137:1742-50.

18. Maurer M, Kaplan A, Rosén K, et al. The XTEND-CIU study: long-term use of omalizumab in chronic idiopathic urticaria. J Allergy Clin Immunol 2018;141:1138-9. 19. Kaplan A, Ledford D, Ashby M, et al. Omalizumab in patients with symptomatic chronic idiopathic/spontaneous urticaria despite standard combination therapy. J Allergy Clin Immunol 2013;132: 101-9.

20. Maurer M, Rosén $\mathrm{K}$, Hsieh H-J, et al. Omalizumab for the treatment of chronic idiopathic or spontaneous urticaria. N Engl J Med 2013;368:924-35.

21. Saini SS, Bindslev-Jensen C, Maurer $\mathrm{M}$, et al. Efficacy and safety of omalizumab in patients with chronic idiopathic/ spontaneous urticaria who remain symptomatic on $\mathrm{H} 1$ antihistamines: a random- 
ized, placebo-controlled study. J Invest Dermatol 2015;135:67-75.

22. Casale TB, Bernstein JA, Maurer M, et al. Similar efficacy with omalizumab in chronic idiopathic/spontaneous urticaria despite different background therapy. J Allergy Clin Immunol Pract 2015;3:74350.

23. Arm JP, Bottoli I, Skerjanec A, et al. Pharmacokinetics, pharmacodynamics and safety of QGE031 (ligelizumab), a novel high-affinity anti-IgE antibody, in atopic subjects. Clin Exp Allergy 2014;44:1371-85. 24. Hollis K, Proctor C, McBride D, et al. Comparison of Urticaria Activity Score over 7 days (UAS7) values obtained from once-daily and twice-daily versions: results from the ASSURE-CSU Study. Am J Clin Dermatol 2018;19:267-74.

25. Hawro T, Ohanyan T, Schoepke N, et al.
Comparison and interpretability of the available Urticaria Activity Scores. Allergy 2018;73:251-5.

26. Stull D, McBride D, Tian $\mathrm{H}$, et al. Analysis of disease activity categories in chronic spontaneous/idiopathic urticaria. Br J Dermatol 2017;177:1093-101.

27. Mathias SD, Crosby RD, Rosén KE, Zazzali JL. The minimal important difference for measures of urticaria disease activity: updated findings. Allergy Asthma Proc 2015;36:394-8.

28. Biagtan MJ, Viswanathan RK, Evan MD, Mathur SK. Clinical utility of the Chronic Urticaria Index. J Allergy Clin Immunol 2011;127:1626-7.

29. Viswanathan RK, Biagtan MJ, Mathur SK. The role of autoimmune testing in chronic idiopathic urticaria. Ann Allergy Asthma Immunol 2012;108:337-41.
30. Bretz F, Pinheiro JC, Branson M. Combining multiple comparisons and modeling techniques in dose-response studies. Biometrics 2005;61:738-48.

31. Pinheiro J, Bornkamp B, Glimm E, Bretz F. Model-based dose finding under model uncertainty using general parametric models. Stat Med 2014;33:1646-61. 32. Türk M, Kocatürk E, Cüre K, Yllmaz İ. Two-week intervals during omalizumab treatment may provide better symptom control in selected patients with chronic urticaria. J Allergy Clin Immunol Pract 2018;6:1389-90.

33. Larenas-Linnemann DES, Parisi CAS, Ritchie C, et al. Update on omalizumab for urticaria: what's new in the literature from mechanisms to clinic. Curr Allergy Asthma Rep 2018;18(5):33.

Copyright @) 2019 Massachusetts Medical Society. 